\title{
¿Qué opinan los universitarios sobre el uso académico de las redes sociales?
}

\section{What do university students think about the academic use of social networks?}

\author{
Patricia Abelairas-Etxebarria \\ Universidad del País Vasco/Euskal Herriko Unibertsitatea \\ patricia.abelairas@ehu.es \\ Jon Mentxaka Arana \\ Universidad del País Vasco/Euskal Herriko Unibertsitatea \\ imentxaka@ehu.es
}

Recibido: 07/02/2019

Aceptado: $05 / 11 / 2019$

Publicado: 01/06/2020

\section{RESUMEN}

Hoy en día, las redes sociales son utilizadas en cualquier momento y en cualquier lugar. Entre los jóvenes, en particular, han supuesto una revolución a la hora de relacionarse. Sin embargo, dichas redes sociales tienen escasa presencia en el aula universitaria para su uso académico.

Este trabajo pretende analizar el uso de las redes sociales de los estudiantes de la Facultad de Economía y Empresa de la Universidad del País Vasco/Euskal Herriko Unibertsitatea. Además, se analiza la opinión de los alumnos y alumnas sobre la posibilidad de introducir las redes sociales en el aula con fines académicos. Con estos objetivos, se ha realizado una encuesta a una muestra de los estudiantes de dicha facultad donde, como principal resultado, se extrae que la gran mayoría de los estudiantes usan habitualmente las redes sociales en el ámbito personal y que están dispuestos a introducir estas redes sociales como herramienta en el aula universitaria.

\section{PALABRAS CLAVE}

Redes sociales; aula universitaria; uso académico.

\section{ABSTRACT}

At the present time, social networks are used anytime and anywhere. Among young people, in particular, they have been a revolution when it comes to socializing. However, these social networks have little presence in the university classroom for an academic use.

This paper aims to analyze the use of social networks of students of the Faculty of Economics and Business of the University of the Basque Country / Euskal Herriko Unibertsitatea. In addition, it analyzes the opinion of the students about the possibility of introducing social networks in the classroom for academic purposes. With these objectives a survey has been carried out on a sample of the students of said faculty where, as the main result, it is extracted that the vast majority of students routinely use social networks in the personal sphere and that they are willing to introduce these social networks as a tool in the university classroom.

\section{KEYWORDS}

Social networks; university classroom; academic use. 


\section{CITA RECOMENDADA}

Abelairas-Etxebarria, P. (2020). ¿Qué opinan los universitarios sobre el uso académico de las redes sociales? Revista Interuniversitaria de Investigación en Tecnología Educativa, 8, 1-12. http://dx.doi.org//10.6018/riite.362121

\section{Principales aportaciones del artículo y futuras líneas de investigación:}

- Conocer el uso de las redes sociales de los universitarios.

- Conocer la opinión de los universitarios del uso de las redes sociales para su uso académico.

- Futura incorporación de las redes sociales en el aula universitaria.

\section{INTRODUCCIÓN}

En los últimos años las redes sociales son utilizadas cada vez por más personas y más frecuentemente. De hecho, podría decirse, que las redes sociales están pasando a ser una de las formas principales para comunicarse, sobre todo entre los jóvenes. Los jóvenes están conectados a las redes sociales y en ellas interactúan, conocen gente y aprenden (Prendes et al., 2015).

El principal objetivo de las redes sociales es el de conectar a unas personas con otras personas, las cuales quieran comunicarse entre ellas, ya sea por amistad o porque comparten interés por algún tema en concreto. Se estructuran como plataformas online donde los usuarios que se registran intercambian mensajes, fotos o archivos de manera inmediata mediante su teléfono, tableta u ordenador. La mayoría de los jóvenes hacen un uso habitual de las redes sociales y las usan, principalmente, para relacionarse con sus iguales y expresar sus opiniones (Colás-Bravo et al., 2013).

Pese al boom del uso de las redes sociales en los últimos tiempos, en especial entre los jóvenes, no se observa la misma evolución en su uso académico. Los estudiantes universitarios están permanentemente conectados mediante su teléfono móvil, en muchas ocasiones, incluso en el aula universitaria, pero siempre para un uso personal y no para un uso académico. El escaso uso didáctico de las redes sociales por parte de los jóvenes estudiantes está causado en gran medida por la escasa importancia que el profesorado y las instituciones les dan a dichas redes sociales como herramienta (Gómez-Aguilar et al., 2012).

Sin embargo, las Tecnologías de la Información y la Comunicación (TIC) son herramientas fundamentales en los cambios en la educación, el ocio, la comunicación o las relaciones sociales (Bringué \& Sádaba, 2009) y cabría pensar que una de estas TIC, como lo son las redes sociales, podrían ser un instrumento para modificar y modernizar las formas de relacionarse en el ámbito académico entre los propios alumnos y entre el profesor y los alumnos. De hecho, las redes sociales ofrecen muchas posibilidades para los docentes para interaccionar con el alumno (Valenzuela, 2013).

En el ámbito educativo no universitario existen investigaciones centradas en los usos de las TIC, y en particular, de las redes sociales con usos docentes. Así, Pérez (2018), estudia el uso de las redes sociales y smartphones en alumnos de Educación Primaria de 11 y 12 años concluyendo que existe un grado de uso significativo de las redes sociales en estas edades. Por otro lado, De Haro (2009) estudia las redes sociales aplicadas a la práctica docente y destaca que éstas son prácticamente no utilizadas con fines estrictamente educativos en la educación preuniversitaria. Siguiendo esta última idea, al revisar la literatura, se ha observado que los estudios sobre otras TIC con fines docentes son más frecuentes que los estudios sobre esos mismos usos centrados en las redes sociales en particular.

En cuanto a estudios que analicen la utilización de las redes sociales en el ámbito universitario, cabe destacar a autores como Santoveña-Casal \& Bernal-Bravo (2019) que analizan la influencia del rol del profesorado sobre la participación social en Twitter y la 
experiencia académica percibida de los estudiantes en la Universidad Nacional de Educación a Distancia. Estos autores concluyen que el uso educativo de las redes sociales motiva más que herramientas tradicionales como los foros, además también concluyen que un rol del profesor que facilite un proceso de aprendizaje independiente es la estrategia adecuada para el uso de las redes sociales en el aula universitaria. Por su parte, Gómez-Hurtado et al. (2018) estudian las concepciones del alumno y profesor sobre Facebook y concluyen que el uso de las redes sociales es un recurso didáctico que promueve y facilita el intercambio colaborativo entre estudiantes y docentes. En esta línea, González et al. (2016) analizan las actitudes de los universitarios hacia el uso educativo de las redes sociales. Como resultados confirman el uso habitual de los estudiantes de las redes sociales en el ámbito personal pero no en el académico, en parte, según este estudio a la falta de iniciativa de los profesores en su uso.

Por otro lado, Prendes et al. (2015) estudian los tipos de uso que hacen los estudiantes universitarios de las redes sociales. Estas autoras confirman el uso lúdico y habitual de las redes sociales de los estudiantes universitarios. También destacan que las herramientas que se utilizan para fines académicos son las que tiene más control de la privacidad. También centrado en este mismo tema, Roses et al. (2013) estudian la diferente forma en la que utilizan las redes sociales los alumnos de ciencias y de letras. Estos autores llegan a la conclusión de que los estudiantes de ciencias son menos favorables al uso de estas herramientas en el aula universitaria, observando, además, que son estos alumnos los que usan menos las redes sociales en el ámbito académico en los casos en los que se les presenta la ocasión. Túñez \& Sixto (2012) estudian el uso de las redes sociales en el aula universitaria y concluyen que las redes sociales deben ser entendidas como un complemento de la docencia, teniendo en cuenta que los alumnos las valoran positivamente como un entorno donde reflexionar y aprender.

Por otro lado, Islas \& Carranza (2011) estudian el uso de las redes sociales como estrategias de aprendizaje, mediante la realización de una encuesta a los alumnos del Centro Universitario de los Altos de la Universidad de Guadalajara de México, y obtienen como resultado que las redes sociales pueden ser utilizadas como estrategia de aprendizaje por los estudiantes. Otros autores, como García (2009), estudian la manera en la que el docente puede potenciar su uso, destacando que el profesor debe adquirir el rol de guía en el uso de estas redes sociales como herramienta docente en el aula universitaria. Por último, Esteve (2009) estudia la relación de la evolución de las TIC con el entorno de Bolonia en la universidad, concluyendo que las universidades españolas, al adaptarse al Espacio Europeo de Educación Superior, deben avanzar en la modernización de sus metodologías educativas.

\section{OBJETIVOS}

El presente estudio busca analizar el uso de las redes sociales de los universitarios y su opinión sobre la introducción de dichas redes en el aula con fines académicos. Se concretaron dos objetivos específicos a llevar a cabo en el presente trabajo:

En primer lugar, analizar el uso de las redes sociales de los estudiantes de la Facultad de Economía y Empresa de la Universidad del País Vasco/Euskal Herriko Unibertsitatea. Se trata de analizar si las utilizan, cómo es su uso, cuánto las utilizan, con qué fines...

En segundo lugar, se trata de analizar la perspectiva de dichos estudiantes universitarios sobre la posibilidad de introducir las redes sociales en el aula universitaria como herramienta de aprendizaje y en qué formato las introducirían.

\section{METODOLOGÍA}

Para la consecución de los objetivos detallados anteriormente, se ha llevado a cabo un análisis cuantitativo descriptivo mediante la técnica de encuesta en la Facultad de Economía y Empresa de la Universidad del País Vasco/Euskal Herriko Unibertsitatea, que es la población que se va a analizar. Para la consecución de dicho análisis se ha tomado una muestra aleatoria de 105 alumnos y alumnos que personalmente han contestado a dicho cuestionario en el primer cuatrimestre del año 2016. 
La citada encuesta se diseñó ad hoc teniendo presentes los objetivos de este estudio. Siguiendo los dos objetivos planteados anteriormente, se incluyeron preguntas, en primer lugar, sobre el uso que tienen los estudiantes de las redes sociales, incluyendo cuestiones sobre cuáles son las redes que usan, con qué frecuencia y para qué las usan. En segundo lugar, se incluyeron preguntas para recoger la opinión de los alumnos y alumnas sobre el uso académico de las redes sociales, preguntándoles qué opinan sobre su introducción en el aula, para qué las utilizarían y cuál creen que puede ser la más apropiada para fines académicos. Cabe destacar, que al diseñar la encuesta se introdujeron las principales 3 redes sociales definidas como tal de una manera estricta, para ver así el perfil general de uso de los estudiantes. Sin embargo, los encuestados, en la última pregunta, diseñada como una pregunta abierta, incluyeron la red WhatsApp, la cual no había sido incluida en el resto de preguntas cerradas debido a la falta de consenso sobre su pertenencia a las redes sociales como tales.

\section{RESULTADOS}

Una vez realizada la encuesta se analizaron los datos obtenidos de ella mediante tablas y gráficos que han ayudado a obtener los principales resultados que se presentan a continuación.

Como se puede observar en la Figura 1, la mayoría de los estudiantes que han participado en el estudio tienen 21 años. No obstante, la muestra engloba todas las edades presentes en el estudiantado de grado de la Facultad de Economía y Empresa de la Universidad del País vasco/Euskal Herriko Unibertsitatea, esto es, desde los 19 hasta los 26 años.

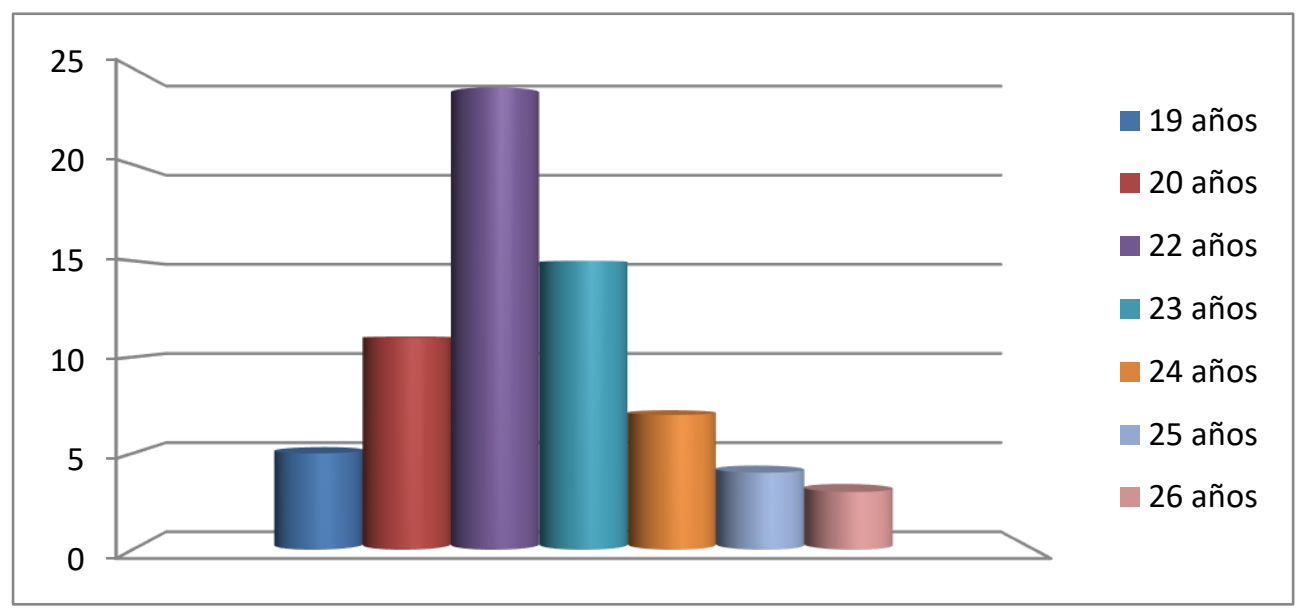

Figura 1. Edad de la muestra utilizada de estudiantes universitarios.

Fuente: Todas las figuras han sido elaboradas por los autores a partir de los resultados obtenidos de la encuesta.

En la Figura 2 se representa la respuesta a la pregunta sobre el conocimiento de los estudiantes de las diferentes redes sociales. Como se muestra, y como cabía esperar, los alumnos tienen un muy alto conocimiento de las redes sociales en general. En concreto, el $100 \%$ de los alumnos conocen las redes sociales Facebook, Twitter y Linkedin. 


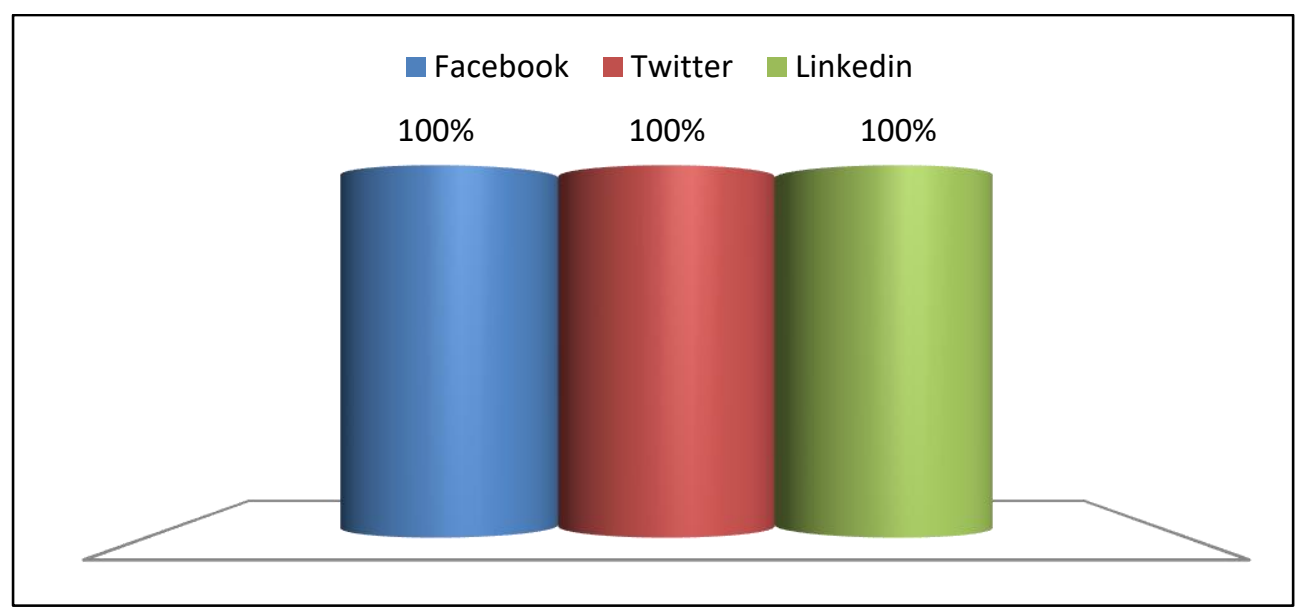

Figura 2. Redes sociales que conocen los estudiantes universitarios.

En la Figura 3 se recogen los datos en cuanto a las redes sociales preferidas por los alumnos. Aparece en primer lugar la red social Facebook con un $84 \%$, seguida de Twitter (64\%) y Linkedin (50\%).

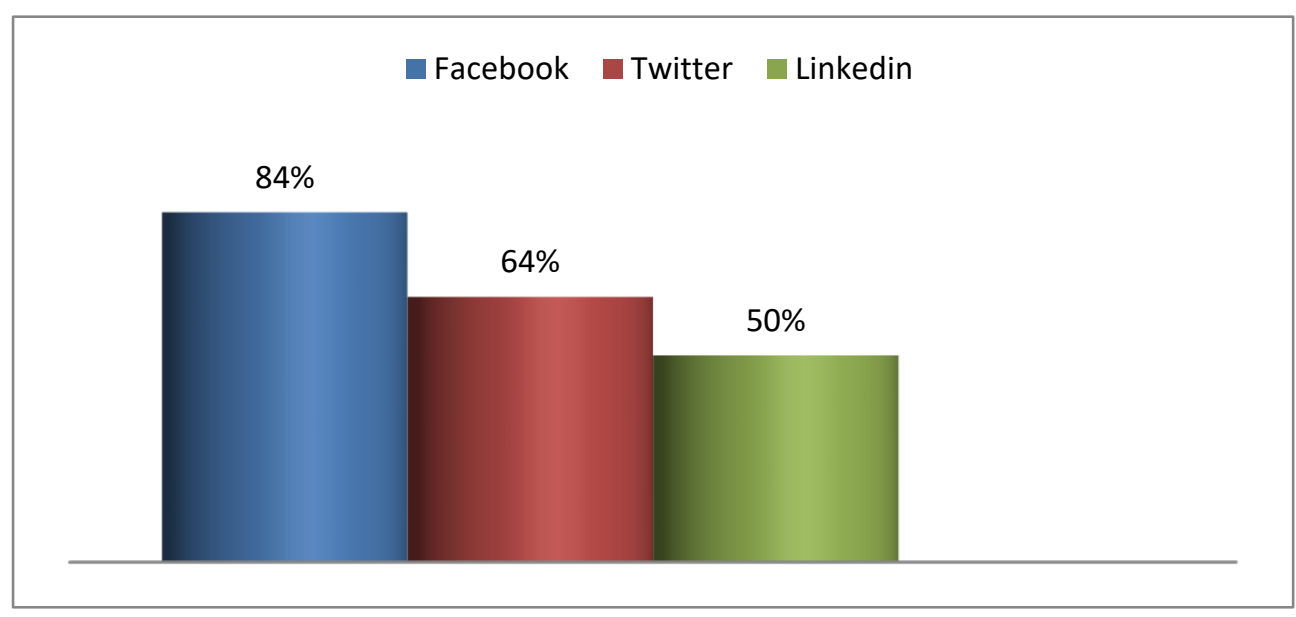

Figura 3. Redes sociales que utilizan los estudiantes universitarios.

Al analizar las respuestas sobre el tipo de uso que le dan a las redes sociales, como muestra la Figura 4, se puede observar que el uso "Buscar amistades nuevas" junto con "Informarse sobre las noticias del día" son los más frecuentes con un $66 \%$ y $65 \%$, respectivamente. A estos, les siguen "Comentar fotos y vídeos" (55\%), "Quedar y relacionarse con sus amigos" (24\%), "Estudiar y aclarar dudas de diferentes asignaturas" (24\%) y "Jugar" (1\%). Más allá de los usos lúdicos, cabe destacar el alto uso que le dan a las redes sociales para recibir información leyendo las noticas y el uso significativo que hacen de las redes sociales para aclarar dudas académicas con los compañeros. Este hecho, hace deducir que es frecuente que los estudiantes de una asignatura creen un grupo en alguna red social para relacionarse entre ellos, lo cual podría ser el primer paso de la creación de un grupo en el que también estuviera incluido el profesor, dotándole de un perfil más académico y más formal. 


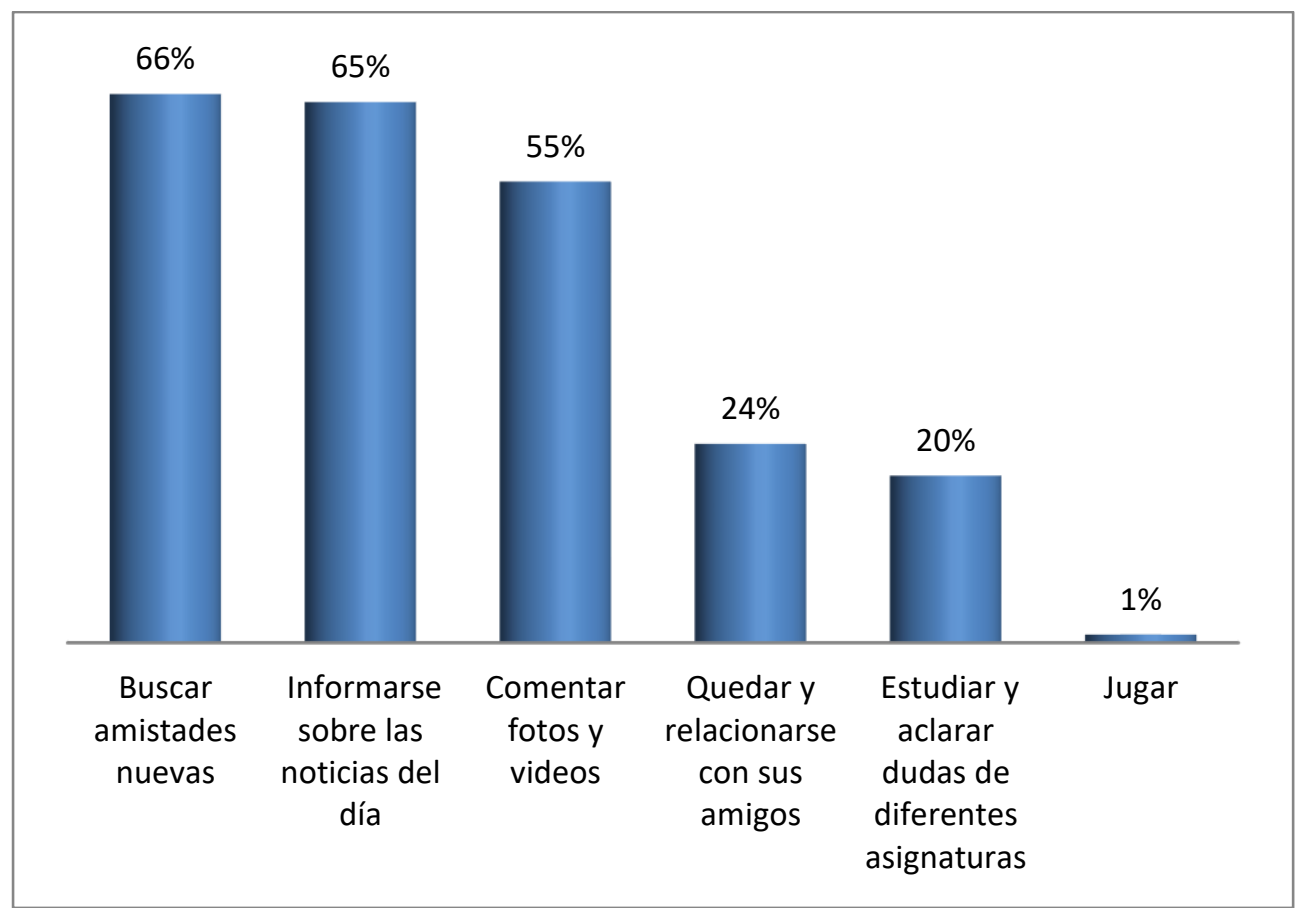

Figura 4. ¿Para qué utilizan los estudiantes universitarios las redes sociales?

La Figura 5 recoge la frecuencia con la que los estudiantes universitarios usan las redes sociales. El $77 \%$ de ellos usan las redes sociales a diario o casi a diario, es decir, tan solo el $23 \%$ lo usan de manera menos frecuente. Además, vista la evolución de las redes sociales en los últimos años en la sociedad en general, el porcentaje de jóvenes y, por tanto, de alumnos que utilizan las redes sociales de manera habitual va en claro aumento. Como se puede observar, el número de alumnos que no tienen contacto con las redes sociales es de un $10 \%$ del total de los estudiantes encuestados.

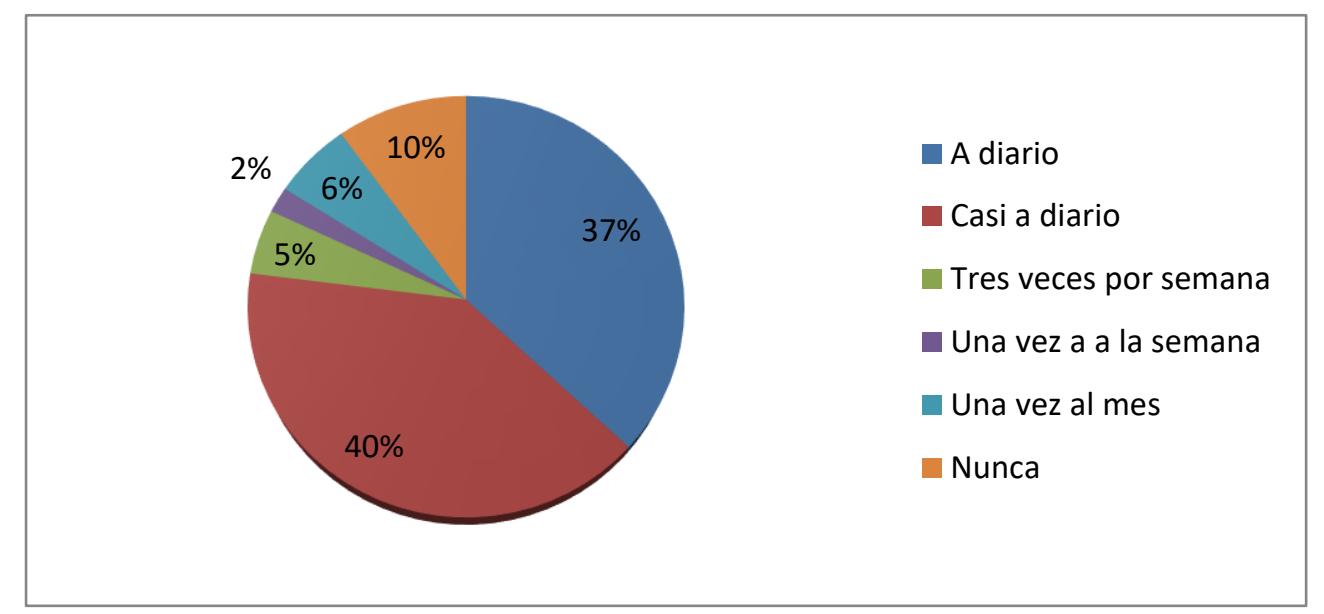

Figura 5. Frecuencia con la que usan las redes sociales los estudiantes universitarios.

Siguiendo en la línea de la Figura 5, la Figura 6 recoge cuál es la opinión de los estudiantes sobre la posibilidad de introducir una red social en el aula con fines académicos. La gran mayoría de ellos, concretamente el $81 \%$, están a favor de utilizarlas como herramienta en el aula universitaria. Este resultado es muy positivo ya que la actitud de la mayoría de los estudiantes es favorable. 


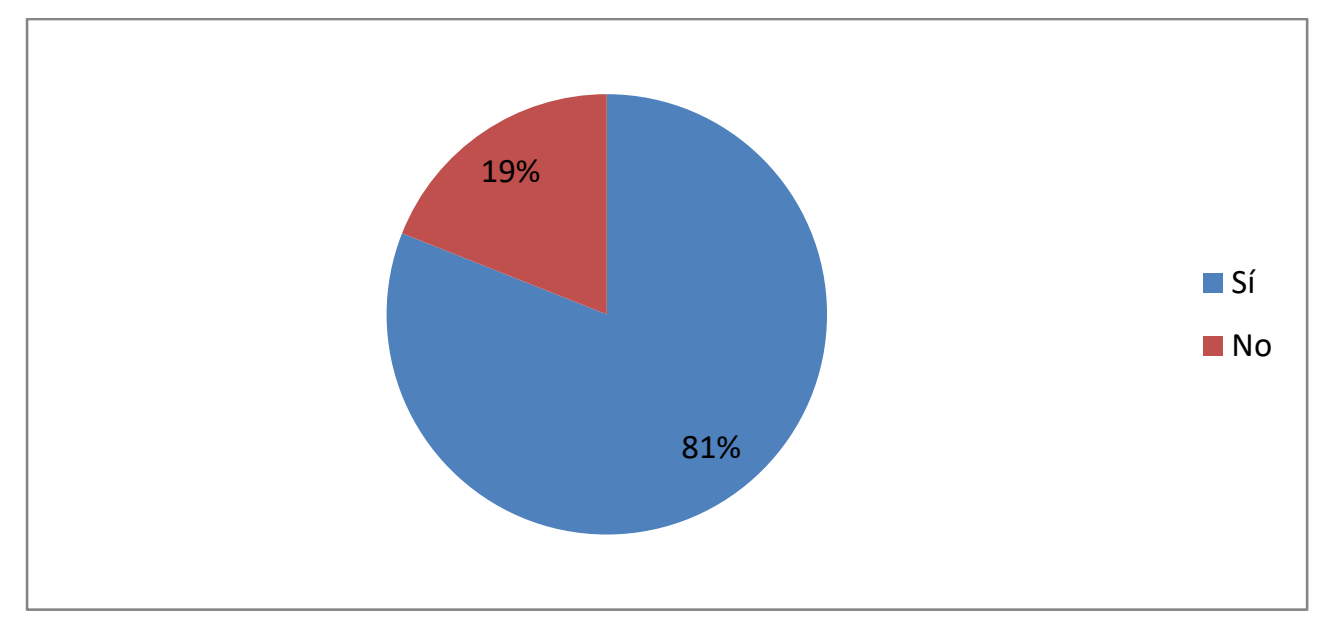

Figura 6. ¿Estás a favor de utilizar una red social con los compañeros y con el profesor?

Tratando de saber más sobre la perspectiva de los estudiantes sobre un escenario en el que una red social fuese utilizada con fines académicos, la Figura 7 recoge cuáles serían los potenciales usos que los alumnos opinan que les darían. El uso principal que le darían a esa potencial red social sería el de intercambiar apuntes, con un $81 \%$. También la usarían para resolver dudas con el profesor en un 56\%, para resolver dudas con los compañeros en un $33 \%$, para compartir opiniones con los compañeros en un 14\%, para compartir documentos en un $11 \%$ y para compartir opiniones con el profesor en un $11 \%$.

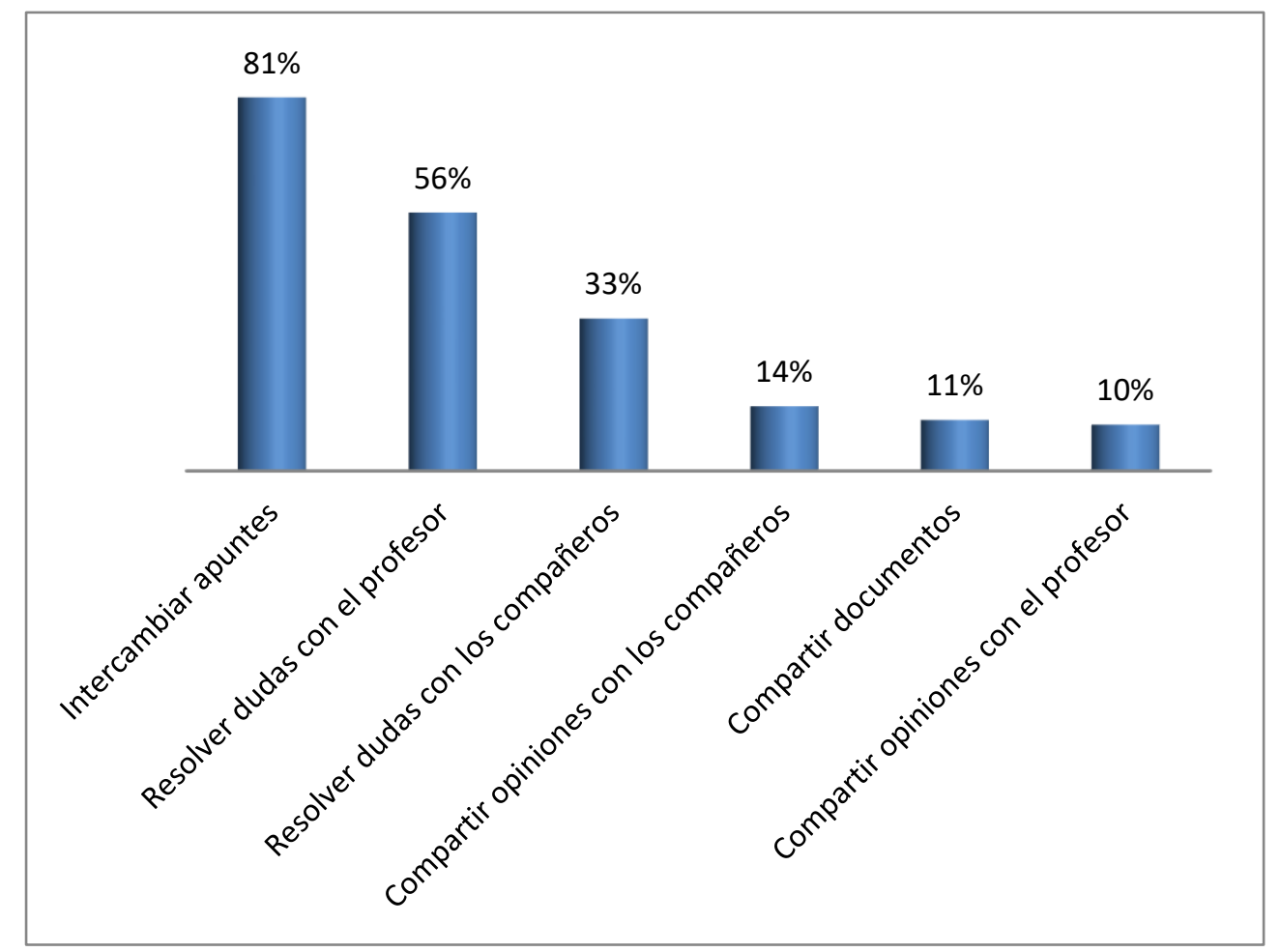

Figura 7. ¿Para qué utilizarías un grupo de una asignatura creado en una red social?

Finalmente, la Figura 8 recoge cuál sería la red social más adecuada para llevar a cabo el objetivo según los universitarios. El 78\% opinan que Facebook sería la idónea para dicha tarea, siendo ésta, como se ha citado anteriormente, la red social más conocida y más utilizada. Sin embargo, el $22 \%$ de los encuestados creen que la aplicación Facebook es la más adecuada para dicho fin. Cabe destacar que WhatsApp no ha sido tenida en cuenta en el resto de la encuesta, ya que, en principio, no ha sido considerada red social al no haber consenso general en incluir este servicio de mensajería dentro de las redes sociales. Sin embargo, puede ser de 
interés saber que algunos alumnos opinan que podría ser interesante usarla para comunicarse con los demás alumnos y con el profesor.

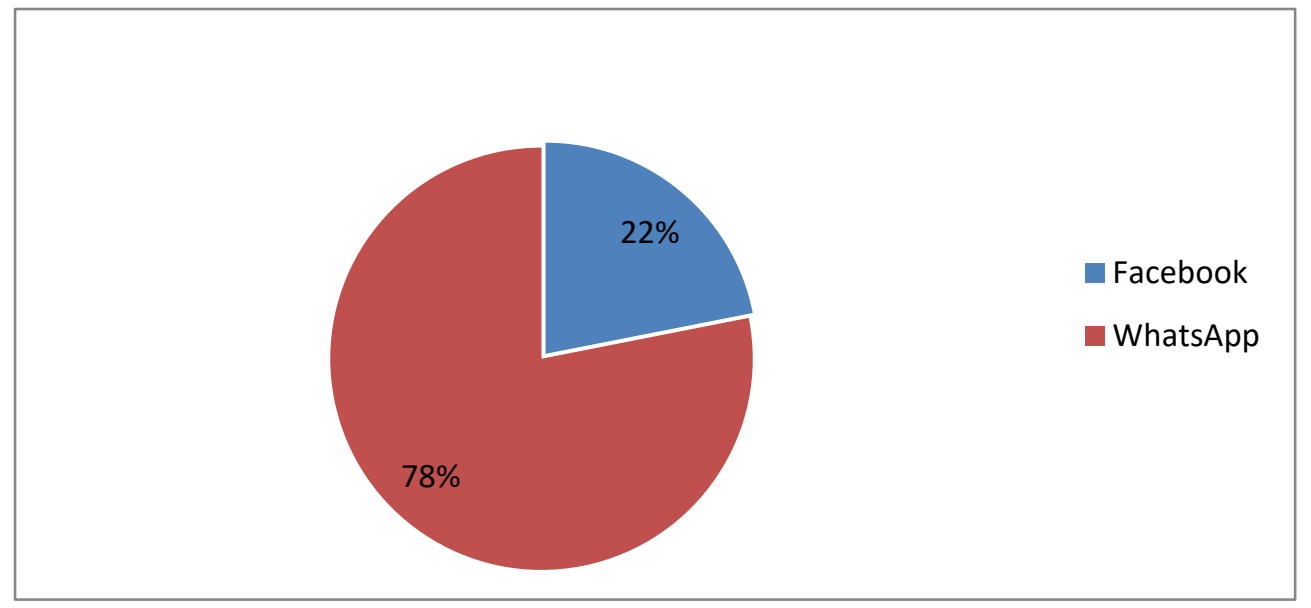

Figura 8. ¿Qué red social utilizarías para este uso académico?

\section{CONCLUSIONES}

En cuanto al primer objetivo de esta investigación, el análisis del uso de las redes sociales de los universitarios de la Facultad de Economía y Empresa de la Universidad del País Vasco/Euskal Herriko Unibertsitatea, se puede afirmar que la totalidad de los jóvenes universitarios conocen las redes sociales y que, la gran mayoría, las utilizan a diario. Es destacable que, en general, no son utilizadas con fines académicos. No obstante, es subrayable el uso significativo (un 20\%) que hacen de las redes sociales para aclarar dudas académicas con otros compañeros. Los principales fines con que son utilizadas son: la búsqueda de nuevas amistades, informarse mediante las noticias, y comentar fotos y videos.

En cuanto al segundo objetivo del presente trabajo, el de analizar la opinión de los universitarios sobre la posibilidad de introducir las redes sociales en el aula universitaria como herramienta de aprendizaje, se confirma que sí estarían dispuestos a su utilización en el aula. En el caso de tener la oportunidad los estudiantes las utilizarían para intercambiar apuntes en su mayoría y también en gran medida para resolver dudas con el profesor. Por último, como red social a utilizar para un uso académico proponen, en su mayoría, la red social Facebook.

Vistos los resultados de este análisis, cabría pensar que si los estudiantes universitarios no utilizan las redes sociales como herramienta en el aula es debido a que no se les brinda esa oportunidad, en general, en las aulas universitarias.

Se podría decir que el mundo digital y las TIC se han convertido en una herramienta necesaria en todos los ámbitos. La sociedad actual ha internalizado estas nuevas tecnologías y, en concreto, los estudiantes en la universidad están preparados para llevar esta tecnología al mundo académico. Está en las manos de los docentes la oportunidad de utilizar herramientas del siglo XXI en el desarrollo de los estudiantes de grado.

Sería interesante, además, no olvidar que la implementación de estos nuevos métodos puede presentar ciertas dificultades entre algunos profesores y una minoría de alumnos, las cuales serían necesarias resolver mediante la oferta de herramientas y formación diseñadas a tal efecto.

\section{REFERENCIAS BIBLIOGRÁFICAS}

Bringué, S. \& Sádaba, C. (2009). La generación interactiva en España. Niños y adolescentes ante las pantallas. Barcelona: Ariel, Colección Fundación Telefónica. https://dadun.unav.edu/bitstream/10171/18443/1/n15-sadaba-chalezquer.pdf 
Colás-Bravo, P., González-Ramírez, T. \& de Pablos-Pons, J. (2013). Juventud y redes sociales: Motivaciones y usos preferentes. Revista Comunicar, 20, 15-23. https://doi.org/10.3916/C40-2013-02-01

De Haro, J. (2009). Las redes sociales aplicadas a la práctica docente. DIM: Didáctica, Innovación y Multimedia, 13. https://www.raco.cat/index.php/DIM/article/view/138928

Esteve, F. (2009). Bolonia y las TIC: de la docencia 1.0 al aprendizaje 2.0. La Cuestión Universitaria, 5, 58-67.

http://polired.upm.es/index.php/lacuestionuniversitaria/article/viewFile/3337/3402

García Sans, A. (2008). Las redes sociales como herramienta en el aprendizaje colaborativo: una experiencia con Facebook. Periodismo, Comunicación y Sociedad, 2, 49-59.

Gómez-Aguilar, M., Roses-Campos, S. \& Farias-Batlle R. (2012). El uso académico de las redes sociales en universitarios. Revista Comunicar, 19, 131-138. https://doi.org/10.3916/C38-2012-03-04

Gómez-Hurtado, I., García Prieto, F.J. \& Delgado-García M. (2018). Uso de la red social Facebook como herramienta de aprendizaje en estudiantes universitarios: estudio integrado sobre percepciones. Perspectiva Educacional, 57, 99-119. http://www.perspectivaeducacional.cl/index.php/peducacional/article/view/645

González Martínez, J., Lleixà Fortuño, M. \& Espuny Vidal, C. (2016). Las redes sociales y la educación superior: las actitudes de los estudiantes universitarios hacia el uso educativo de las redes sociales, de nuevo a examen. Education in the Knowledge Society 17, 21-38. https://revistas.usal.es/index.php/eks/article/view/eks20161722138

Islas Torres, C. \& Carranza Alcántar, M. (2011). Uso de las redes sociales como estrategias de aprendizaje. ¿Transformación educativa? Apertura. Revista de innovación educativa, 3. http://www.udgvirtual.udg.mx/apertura/index.php/apertura/article/view/198/213.Mexico.con $\underline{\text { sultado }}$

Pérez Escoda, A. (2018). Uso de smartphones y redes sociales en alumnos/as de Educación Primaria. Revista Prisma Social, 20, 76-91. https://revistaprismasocial.es/article/view/2310

Prendes Espinosa, M.P., Gutiérrez Porlán I. \& Castañeda Quinteros, L. (2015). Perfiles de uso de redes sociales: estudio descriptivo con alumnado de la Universidad de Murcia. Revista Complutense de Educación 175, 26, 175-195. https://revistas.ucm.es/index.php/RCED/article/view/46439

Roses, S., Gómez Aguilar, M. \& Farias, P. (2013). Uso académico de redes sociales: análisis comparativo entre estudiantes de Ciencias y de Letras. Historia y Comunicación Social,18, 667-678. https://core.ac.uk/download/pdf/38816722.pdf

Santoveña-Casal, S. \& Bernal-Bravo C. (2019). Explorando la influencia del docente: Participación social en Twitter y percepción académica. Comunicar, 58, 5-84. https://doi.org/10.3916/C58-2019-07

Túñez López, M. \& Sixto García, J. (2012). Las redes sociales como entorno docente: análisis del uso de Facebook en la docencia universitaria. Pixel-Bit. Revista de Medios y Educación, 41, 77-92.

Valenzuela Argüelles, R. (2013). Las redes sociales y su aplicación en la educación. Revista Digital Universitaria, 14 (4). http://www.revista.unam.mx/vol.14/num4/art36/art36.pdf 


\title{
INFORMACIÓN SOBRE LOS AUTORES
}

\section{Patricia Abelairas-Etxebarria}

Universidad del País Vasco/Euskal Herriko Unibertsitatea

Profesora en el Departamento de Economía Aplicada V de la Facultad de Economía y Empresa de la Universidad del País Vasco/Euskal Herriko Unibertsitatea. Docente de diversas asignaturas como Introducción a la Microeconomía, Introducción a la Macroeconomía, Informática y Sistemas de Información y Gestión Empresarial. Las principales áreas de investigación abarcan el uso de las redes sociales en la universidad, el mercado del suelo y de la vivienda y la econometría espacial aplicada.

https://www.ehu.eus/eu/web/economiaplicada5/patricia-abelairas-etxebarria

\section{Jon Mentxaka Arana}

Universidad del País Vasco/Euskal Herriko Unibertsitatea

Graduado en Administración y Dirección de Empresas. En la actualidad, aprovisionador de compras en el Departamento de Compras de la empresa Chavesbao situada en Larrabetzu (Bizkaia).

https://es.linkedin.com/in/jon-mentxaka-arana-a5355582$$
\text { (c) }
$$

Los textos publicados en esta revista están sujetos a una licencia de Reconocimiento 4.0 España de Creative Commons. Puede copiarlos, distribuirlos, comunicarlos públicamente y hacer obras derivadas siempre que reconozca los créditos de las obras (autoría, nombre de la revista, institución editora) de la manera especificada por los autores o por la revista. La licencia completa se puede consultar en:Licencia Creative Commons Atribución-NoComercial-Compartir por igual 4.0 Internacional.
\end{abstract}




\section{Anexo}

A continuación, se adjunta el formato de encuesta aplicada durante el estudio:

1-Edad $\square$
2- Curso $\square$ - Redes sociales que conoces:
a) Facebook
b) Linkedin
c) Twitter

4- Redes sociales que usas:
a) Facebook
b) Linkedin
c) Twitter

5- Frecuencia con la que usas las redes sociales:
a) Todos los días varias veces
b) Casi todos los días
c) 3 veces por semana
d) 1 vez por semana
e) 1 vez al mes
f) Nunca

6- ¿Para qué usas las redes sociales?
a) Quedar y relacionarte con tus amigos
b) Informarte sobre las noticias del día
c) Cotillear y buscar amistades nuevas
d) Comentar fotos y videos
e) Para estudiar y aclarar dudas de diferentes asignaturas
f) Para jugar
g) Otras

7- ¿Qué te parecería introducir la redes sociales en la enseñanza universitaria: (Escala del 1= en contra, al $5=$ totalmente de acuerdo)

8- ¿Te gustaría poder usar las redes sociales para intercambiar información de las asignaturas que cursas?

$$
\begin{aligned}
& \text { Sí } \\
& \text { No }
\end{aligned}
$$

9- ¿Qué te parecería la posibilidad de resolver dudas con el profesor mediante las redes sociales?: (Escala del $1=$ mal, al $5=$ bien)

10- ¿Para qué utilizarías un grupo de una asignatura creado en una red social?:
a) Resolver dudas con los compañeros.
b) Resolver dudas con el profesor.
c) Compartir documentos. 
d) Compartir fotografías.

e) Compartir opiniones con los compañeros.

f) Compartir opiniones con el profesor.

11- ¿Qué red social crees que sería la idónea para esa práctica?
a) Facebook
b) Linkedin
c) Twitter
d) WhatsApp 